\title{
Fans und Vergemeinschaftungsprozesse
}

\section{Thorsten Berndt}

Bettina Fritzsche, Pop-Fans - Studie einer Mädchenkultur. 2. Auflage, Wiesbaden: VS 2011, 305 S., br., $29,95 €$

Jonas Gabler, Die Ultras - Fußballfans und Fußballkulturen in Deutschland. 5. Erw. Auflage, Köln: PapyRossa 2010, 249 S., br., 14,90€

Sven Kathöfer / Jochem Kotthaus (Hrsg.), Block X - Unter Ultras. Ergebnisse einer Studie über die Lebenswelt Ultra in Westdeutschland. Weinheim: Beltz Juventa 2013, 284 S., br., $24,95 €$

Daniel Langer, Faszination Ultras - Aspekte und Erklärungsansätze zur Fußballfan- und Jugendkultur. Bonn: Scienta Bonnensis 2010, 92 S., br., 16,90€

Thomas Nern, Rezeption von Sportveranstaltungen durch Fans. Eine empirische Exploration erlebnis- und verhaltensbezogener Faktoren und ihrer soziologischen Rahmenbedingungen am Beispiel von Spielen der Fußball-Bundesliga. Frankfurt a. M.: Lang 2011, 234 S., gb., 41,80€

Yvonne Niekrenz, Rauschhafte Vergemeinschaftungen - Eine Studie zum rheinischen Straßenkarneval. Wiesbaden: VS 2011, 290 S., br., 34,95€

Jochen Roose / Mike S. Schäfer / Thomas Schmidt-Lux (Hrsg.), Fans. Soziologische Perspektiven. Wiesbaden: VS 2010, 442 S., br., 34,95€

Almut Sülzle, Fußball, Frauen, Männlichkeiten - Eine ethnographische Studie im Fanblock. Frankfurt a. M.: Campus 2011, 395 S., br., 34,90€

Schlüsselwörter: Vergemeinschaftung - Fans - Ultras

Das Phänomen Fans ist spätestens seit der WM 2006 aus der Schmuddel-Ecke in die gesellschaftliche Mitte vorgedrungen und erfreut sich einer allgemeinen Popularität. Fan ist ein Begriff des Alltags und kein Fachbegriff - er bezeichnet etwas, was wir alle kennen. Er hat also seine eigene empirisch-phänomenale oder alltägliche Realität und keinen theoretischen, begrifflich-kohärenten Ursprung. Dieser daraus hervorgehenden prinzipiellen Vielfalt soziologisch-theoretischer Perspektiven soll in dieser Sammelbesprechung damit begegnet werden, dass als roter Faden eine gemeinschaftssoziologische Perspektive eingenommen wird. Fans werden in dieser aktuellen Forschung oft im Rahmen der posttraditionalen Vergemeinschaftungsformen gedeutet, besonders mit Hinblick auf ihren juvenilen und eventhaften Charakter (Hitzler und Niederbacher 2010). Sie werden somit einerseits von traditionellen Formen der Gemeinschaft (Tönnies 1991) unterschieden, andererseits von kommunitaristischen Formen (Etzioni 1995), etwa local communities in Städten (Joas 2007), aber auch von intentionalen Gemeinschaften (Grundmann, Dierschke, et al. 2006). Wie schwierig diese begriffliche Trennung am Phänomen Fans zu 
bewerkstelligen ist und was es für neue Erkenntnisse dazu gibt, soll im Folgenden gezeigt werden.

Der umfangreiche Sammelband „Fans. Soziologische Perspektiven“ von Jochen Roose, Mike S. Schäfer und Thomas Schmidt-Lux ist in der Reihe Erlebniswelten des VS-Verlages erschienen, in der Phänomene wie Events, Szenen und Jugendkulturen in den Blick genommen werden. In dem ersten Beitrag geben die Herausgeber dem Leser zunächst eine Definition des Begriffs Fan an die Hand: „Menschen, die längerfristig eine leidenschaftliche Beziehung zu einem für sie externen, öffentlichen, entweder personalen, kollektiven, gegenständlichen oder abstrakten Fanobjekt haben und in die emotionale Beziehung zu diesem Objekt Zeit und/oder Geld investieren“"(12).

Eine von Roose und Schäfer durchgeführte explorative Online-Befragung in Deutschland versucht Hinweise auf die Verteilung und Beschaffenheit des Fanverhaltens zu erkunden. Trotz einschränkender Faktoren ließen sich folgende Aussagen treffen: Ein Fan wende sich mit einigem Aufwand seinem Fanobjekt zu, dessen Begeisterung sei Kern der Beziehung, auch ohne kritiklose Selbstauflösung. Ein Großteil der Freizeit werde dafür verwendet, „die Geschicke des Fanobjektes zu verfolgen, Informationen zu sammeln, sich mit anderen über das Fanobjekt auszutauschen und oftmals auch, das Fanobjekt zu unterstützen oder Zugehörigkeit zu demonstrieren“"(19).

Zum Stand der Forschung kritisieren die Autoren, dass es eine gewisse Einengung auf spezifische Bereiche (z. B. Fußballfans) und Fragestellungen (deviantes Verhalten, Adoleszenz, rituelle Handlungen) gebe. Ebenso existiere kaum quantitative Forschung mit repräsentativem Anspruch und es gebe eine Theorieabstinenz in hypothesentestender und -generierender Richtung. Der Sammelband möchte zu diesen Punkten - die meiner Meinung nach zutreffend sind - einen positiven Beitrag leisten.

Im nächsten Beitrag durchleuchten die Herausgeber den Phänomenbereich Fans in theoretischer Perspektive. Aus der Rational Choice Sicht werden die Auswahlentscheidungen des (werdenden) Fans unter dem Streben nach sozialer Wertschätzung und/oder der Intensivierung körperlichen Wohlbefindens sowie der Erlebnissicherung auf einem prekärem Erlebnismarkt thematisiert. Der Goffmansche Ansatz biete neben der semantischen Nähe von Publikum, Bühne und Akteuren die fruchtbaren Konzepte des face-work und impression-management zur Inszenierung der Fan-Rolle. Im Bourdieuschen Sinne lasse sich schließlich „verstehen, dass mit der Schließung von Fan-Gemeinschaften nach außen interne Strukturierungen und deren Strukturierungslogiken wichtiger werden, die sich als Herausbildung von sub- oder populär-kulturellem Kapital beschreiben lassen“" (41). Das inkorporierte kulturelle Kapital, in Form von Wissen und spezifischen Kompetenzen, nehme in dieser Hinsicht gegenüber der bloßen Akquise objektivierten Kapitals (etwa im Kauf eines Fan-Schals) zu. Im Ergebnis plädieren die Autoren für die stärkere und fundierte Berücksichtigung allgemeiner soziologischer Theorie im Phänomenbereich Fans (41f.).

Thomas Schmidt-Lux macht es sich im dritten Beitrag zur Aufgabe, eine kleine Geschichte der Fans darzulegen. Zum einen geht er der Geschichte des Begriffs Fan nach, der seinen Ausgangspunkt in einer Zeitungsberichterstattung von $1889 \mathrm{zu}$ den „Kansas City baseball fans" in den USA nehme und alsbald zum Allgemeingut im Sportjournalismus gehöre (50). Zum anderen zeigt der Autor auch den historisch weit vorausliegenden Bedeutungsgehalt von Fans im Zusammenhang mit Wettkampf-Publikum auf - seien es nun ägyptische Ringkämpfe, olympische Kämpfe oder Wagenrennen im alten Rom (52f.). Je mehr sich die Rollenaufteilung in Akteure und Publikum festige und die Medien bei der 
Verbreitung an Gewicht zunähmen, desto mehr Bereiche vom Sport bis zum Theater würden erfasst (57). Der steigenden Bedeutung als Massenphänomen käme die Kommerzialisierung unter dem postmodernen Zwang der Differenzierung und Individualisierung der Fankultur anbei. So ließe sich eine zivilisationsgeschichtliche, eine mediengeschichtliche und eine modernisierungsgeschichtliche Fangeschichte zeigen.

Gunnar Otte zielt in seinem Beitrag darauf, Fans unter dem Aspekt der Sozialstrukturanalyse zu betrachten und liefert ein Erklärungsmodell, welches die Sozialstruktur über die Lebensführung mit dem Fantum vermittelt. Im Zentrum stünden dabei die Systemzugehörigkeiten von Fanobjekten, die Homophilie zwischen Fans und Fanobjekten sowie die Variationen von Intensität und Formen des Fantums. Ein Abgleich mit vorhandenen aber nicht passgenauen - Daten zeige ein zunächst heterogenes Bild, in dem z. B. Bourdieus These von klassenstrukturierten Vorlieben für Sportarten nicht konsistent sei (97). So fordert der Autor schließlich auch eine repräsentative empirische Überprüfung seines Erklärungsmodells.

Unter dem Titel Fans und Emotionen versammelt Mike S. Schäfer zunächst eine kleine Bestandsaufnahme einer Soziologie der Emotionen, beginnend mit Simmel. Emotionen werden als Ergebnis sozialer Interaktionen und Strukturen, als kulturelle Kodierung und als Konstruktionsform sozialer Wirklichkeit analysiert. Für eine Soziologie der Fan-Emotionen schließlich, kann eine Verbindung mit faninterner Hierarchisierung, Kommerzialisierung und Vergemeinschaftung gewinnbringend sein.

Im Abschnitt Fans und kulturelle Praxis wird von Rainer Winter zum einen die Veränderung des Verhältnisses von akademischer Kultur zur Fankultur von einem für den Wissenschaftler zu verbergenden Makel hin zu einer autoethnographisch zu nutzenden Ressource thematisiert. Zum anderen wird auf die „intensivierte Verdichtung, Spezialisierung und Differenzierung von Fanwelten“ (163) im Zuge der rasanten Medialisierung hingewiesen, die auch den Begriff der virtuellen Ethnographie für die Forschung attraktiv mache. Abschließend thematisiert Winter das widerständige, Sinn aushandelnde und vorbildhafte Potential der Fankultur für die Orientierung des Mainstreams.

Winfried Gebhardt geht in seinem Beitrag Fans und Distinktion in einer alternativen Begriffsverwendung der Distinktion Bourdieus darauf ein, dass sie „im Kern als eine individuelle und/oder kollektiv vollzogene Abgrenzungshandlung“ (187) zu verstehen sei und somit Vollzugscharakter in der Praxis habe. Um dieses Wie der Distinktionspraxis näher zu analysieren, wählt der Autor die Goffmansche Theorie der Selbstdarstellung und wendet diese auf die Praxis der Fans an. Für den Begriff der dramatischen Gestaltung gelte beispielsweise „das Explodieren der Anspannung in ekstatischem Gekreische“ (196), sobald das Fanobjekt die Bühne betritt. Das Fansein als Praxis der Distinktion in Selbstdarstellung ziehe seine Attraktivität aus der Verbindung der ambivalenten Struktur von Individualität und Sehnsucht nach Gemeinschaft.

In Fans und Gender von Bettina Fritzsche wird das Potential der Fanforschung hinsichtlich dekonstruktiver Ansätze der Geschlechterforschung beleuchtet. Die Autorin kommt zu dem Schluss, dass unter dem Begriff einer Exzess-Forschung die Betrachtung normüberschreitender Aspekte des Fantums (als Empowerment verstanden) die Bedingung für die Infragestellung gesellschaftlicher Machtverhältnisse - wie eben die hierarchische Geschlechterordnung - biete (246).

Alexander Leistners Beitrag Fans und Gewalt zielt in den am besten erforschten Bereich. Der Autor stellt eine fanspezifische Typologie von Gewalt auf: Intervenierende (di- 
rekt aufs Fanobjekt oder unmittelbare Umfeld gerichtet), gesellige (ungerichtet, aber gemeinschaftsstiftend rauschhaft) und kompetitive Gewalt (gegen direkte konkurrierende Fans) und wendet sie auf das empirische Feld der Fußballfanszene an. Im Ergebnis zeigt Leistner, dass damit eskalationsfördernde Bedingungen und innere Dynamiken identifiziert werden könnten, die gegen Verharmlosungserklärungen oder die Reduzierung auf den Inszenierungscharakter sprechen. Gewalt sei „vor allem für interaktionszentrierte Fanszenen typisch und konstitutiver Teil der Gruppenidentität“ (275).

Thomas Schmidt-Lux geht in seinem Beitrag Fans und Religion einer, besonders auch in der öffentlichen Diskussion, beliebten Verbindung nach. Mit dem Begriff der kollektiven Efferveszenz (Durkheim) im Sinne von rauschhaften Vergemeinschaftungsprozessen sowie über das Konzept der Rituale und Symbole, des Heiligen Kosmos (Luckmann), der asketischen und methodischen Lebensführung (Weber) sowie neueren Ansätzen (Religious-Economies-Approach) verbindet der Autor den Phänomenbereich Fans mit religionssoziologischen Ansätzen für weitere fruchtbare Analysen.

Der Beitrag Fans und Konsum von Leila Akremi und Kai-Uwe Hellmann stellt sich zur Aufgabe, die Bereiche der Konsumforschung oder „Brand Community“-Forschung mit dem Konzept Fan zu verbinden. Hierbei sei der Umschwung vom zufriedenen Kunden zum „Raving Fan“ das entscheidende Moment. Ergebnisse eines Forschungsprojektes darlegend, zeigen die Autoren, dass es hinsichtlich der Intensität oder des Fanatismus / Extremismus (325) des Fantums eine deutliche Abschwächung vom Bereich der Sport-, Musik- und Medienfans zu den Markenfans gebe.

Unter dem Aspekt der Partizipation von Fans zeigen Jochen Roose und Mike S. Schäfer, dass Fans - mit leichten Einschränkungen bei Fußballfans - die „Entscheidungen ihrer Fanobjekte selten auf institutionalisiertem (z. B. in Wahlen) oder nicht-institutionalisiertem (z. B. durch Proteste) Wege zu beeinflussen“ suchen (381). Die Autoren kommen zu dem Schluss, dass Fans somit ,auch in der Moderne Anhänger charismatischer Personen(gruppen)“ seien (381) und dass „intensivere Vergemeinschaftungen mit anderen Fans“ offensichtlich „nicht zu mehr politischer Mitwirkung“ führe (382).

Viktoria Schwenzer und Nicole Selmer beschäftigen sich in ihrem Beitrag mit dem Verhältnis von Fans und Migration. So zeigt sich, dass es keine "grundlegende empirische Forschung zu einer migrantischen Fußballkultur in Deutschland“ gebe (391) und dass dies teilweise durch die Auswahl sozialwissenschaftlicher Forschung mitgeprägt werde, die sich auf die (deutsche) Fußballfankultur mit ihrer symbolischen Konnotation und Inszenierung (weiß/männlich/heterosexuell) beschränke (408). Mehrfachzugehörigkeiten lokaler und globaler Art, die Herkunft- und Aufnahmegesellschaft sowie die hybriden Identitäten vermitteln und ausdrücken können, sind im Sinne der „Frage der Anerkennung [...] als eine zentrale Kategorie in der Analyse von Migrationsprozessen“ zu sehen (409).

Der Sammelband schließt mit einem Beitrag von Jochen Roose zum Thema Fans und Globalisierung. Ein stark transnationalisiertes Feld (Sport, Film, Musik, Literatur) würde in dieser Mischung von den Fans generell auch angenommen, wobei aber z. B. die tatsächliche Erhöhung des Anteils ausländischer Spieler im deutschen Fußball nicht im gleichen Maße Resonanz auf den Kicker-Titelblättern finde. Ähnliches ließe sich für den Musikbereich feststellen. Der Autor möchte hierin aber keinen problematischen Nationalismus erkennen, da die generelle Transnationalität im Fanbereich sehr ausgeprägt sei (433).

Die Herausgeber haben es unter dem Oberbegriff Fan geschafft, die gewisse Einengung auf spezifische Bereiche (wie Fußballfans oder Fans von Popmusik) durch einen 
allgemeineren Zugang zu erweitern, stärker auch Möglichkeiten für quantitative Untersuchungen mit repräsentativem Anspruch aufzuzeigen (die die bestehenden qualitativen Studien gewinnbringend nutzen) und die theoretischen Fokussierungen auf Fragestellungen in Richtung deviantes Verhalten, der Phase der Adoleszenz und den rituellen Elementen des Fanhandelns deutlich aufzubrechen. Das ist ein Gewinn und macht die engere Fan-Forschung deutlich anschlussfähiger, wenn man folgendes berücksichtigt: Gerade unter dem Aspekt, ein möglichst breites Spektrum von theoretischen, thematischen und empirischen Anknüpfungspunkten zum Konzept Fan aufzuzeigen, kommt die Frage auf, was denn z. B. mit der sozialkonstruktivistischen oder der ethnomethodologischen Sichtweise zu gewinnen wäre - wenn man den Begriff Fan und Fanobjekt als ein sich gegenseitig in Existenz bringendes, wechselseitig abhängiges Interaktionsphänomen sieht. Des Weiteren engt die tendenziell abgrenzende Stellungnahme gegen eine "Szene“- oder „urban tribes“-Deutung (42) ohne argumentative wissenschaftliche Diskussion den als weit proklamierten Blick leider wieder ein. Das entspricht dem generellen Hang des Sammelbandes, eher einen erklärenden Zugang zum Phänomen finden zu wollen.

Bei dem Werk „Pop-Fans - Studie einer Mädchenkultur“ von Bettina Fritzsche handelt es sich um die zweite, weitestgehend unveränderte Auflage des bereits 2003 erschienenen Werkes in der Reihe Geschlecht und Gesellschaft des VS-Verlages, weshalb das Werk hier auch nicht in seiner Breite besprochen wird. Die Autorin untersucht weibliche Fans von Boy- und Girlgroups in Hinsicht auf geschlechts- und identitätsbezogene Praktiken bzw. Kulturen. Im Rahmen medien- und handlungstheoretischer Ausgangspunkte (Sue Wise), die auch die gender- und psychoanalytische Ausrichtung beachten, nimmt die Autorin gemäß dem theoretical sampling insgesamt 19 narrative Interviews und drei Gruppeninterviews mit weiblichen Pop-Fans zwischen 10 und 18 Jahren vor. Die Analysen erfolgen gemäß der dokumentarischen Methode (Ralf Bohnsack) und zielen im Ergebnis auf Fallbeschreibungen - methodologisch und methodisch verortet sich die Autorin damit im rekonstruktiven Paradigma qualitativer Sozialforschung.

Kennenlernen und Annähern an heterosexualitätsgeprägte romantische Liebe und spätere erotische Leidenschaft, vergemeinschaftende und distinguierende Normverhandlungen der Pop-Fans auch durch mimetisch-körperliche Aktionen des Nach-Tanzens sowie habitualisierte gruppenstabilisierende Beziehungspraktiken durch fankulturelles Engagement sind die wesentlichen Konzepte, die sich in den Analysen zeigen. Im Zentrum der Ergebnisse steht sodann die Erkenntnis, dass die mediale Vermittlung von normativen Folien zwar einen wesentlichen Rahmen darstelle, die kreative Aushandlung und experimentell-praktische Aneignung von geschlechts- und identitätsbezogenen Normen allerdings eine Fan-Peergroup-interne Eigendynamik und -charakteristik besitze.

In der Gegenüberstellung konjunktiver und disjunktiver Beziehungsbewegungen (Karl Mannheim) als gleichsam konstitutiv für Pop-Fans lassen sich die von Bettina Fritzsche konzeptualisierten Fanpraktiken und medienbezogene Ansätze auch für gemeinschaftssoziologische Fragen fruchtbar machen. Eine disjunktive Beziehungsbewegung ist zumindest in der klassischen Beschäftigung mit Vergemeinschaftung ein Zerfallsargument selbiger. Hier gilt es in Zukunft verstärkt auch bei anderen Fan-Gegenstandsbereichen dezidiertere empirische Analysen zu tätigen, die auf mögliche Verschränkungen begrifflich antagonistischer Prozesse achten.

Thomas Nern legt mit seiner Studie „Rezeption von Sportveranstaltungen durch Fans" eine empirische Forschung vor, die sich vor allem auf die Unterschiede zwischen der 
emotionalen Aktivierung bei Betrachtern von Fußballspielen vor dem Fernsehen (Sportsbar) einerseits und direkt im Stadion andererseits bezieht.

Methodisch geht der Autor über zwei hypothesentestende Zugänge vor: Zum einen mittels einer Messung von Selbstauskünften mithilfe eines Semantischen Differentials als „ein Verfahren zur semiprojektiven Erkundung von Affektkonfigurationen“ (194), zum anderen über die Kodierung von Gruppeninterviews als ein stärker an den subjektiven Relevanzen orientiertes Verfahren. Die interdisziplinäre Medien-Rezeptionsforschung kombiniert Verfahren, um die Bandbreite von Emotionen abzudecken: Von Messung bewusster Emotionen z. B. mithilfe von Gruppendiskussionen, bis hin zu physiologischen und (semi-)projektiven Messverfahren für die unbewussteren Emotionen (58). Starke Faktoren für das Aktivierungspotential von Rezeptionsleistungen sollten dabei ausgleichend berücksichtigt werden: Der Ausgang des Fußballspiels (Erfolg oder Misserfolg der eigenen Mannschaft) und der Spielverlauf (Niveau des Spiels der eigenen Mannschaft). Für die Auswertung des Semantischen Differentials wurde eine Faktorenanalyse als exploratives statistisches Verfahren verwendet, die Analyse der Gruppeninterviews war kategorienbildend um die in den Hypothesen aufgestellten Schwerpunkte herum (110).

Thomas Nern kommt zu dem Schluss, dass der direkte Besuch von Sportveranstaltungen im Stadion eine stärkere emotionale Aktivierung zur Folge habe, als die Verfolgung desgleichen vor dem Fernseher in einer Sportsbar. So bestehe der Reiz eines Stadionbesuches ,insbesondere in der aktiven Suche nach hohem Arousal, verbunden mit einem eindeutigen Valenzerleben“ (198). Auch wenn es beträchtliche Parallelen gibt, sind „die Diskrepanzen in der jeweiligen Wirkungsintensität markant“ (202).

Interessant an diesem Befund ist der hypothesentestende und disziplinübergreifende empirische Ansatz, der die zumeist „nur“ qualitativ gewonnenen Forschungseinsichten bestätigt bzw. erweitert. Wesentliche vergemeinschaftende Aspekte wie die konkrete Praxis einer in-group/out-group Konstruktion und die Rituale, auf die die erhöhte emotionale Intensität verweisen könnte, finden verstärkt im Stadion statt und eher weniger in der Sportsbar. Das wiederum gibt ein Argument für ethnographisch-empirische Zugänge, wie wir einen im folgenden Beitrag „Fußball, Frauen, Männlichkeiten - Eine ethnographische Studie im Fanblock“ von Almut Sülzle sehen werden.

Ein stilistisch gelungener Aufhänger, der an H. M. Miners Nacirema (Miner 1956) erinnert, zieht den Leser sofort in den Bann: Die Autorin Almut Sülzle verfremdet die FanWelt geschickt, indem typisch weiblich konnotierte Praktiken im Fanverhalten (z. B. „Männer weinen nicht in der Öffentlichkeit“) aufgezeigt und deren unmännlicher Gehalt mit einer die Arbeit durchziehenden Frage nach dem, was den Fußball und den Fan eigentlich männlich macht, verbunden wird (14f.). Mit diesem Auftakt ist bereits der Plot gelegt: Frauen in Männerdomänen aus Sicht der Frauen selbst zu erforschen.

Zum einen von der ethnographischen Neugier, zum anderen von der Genderforschung bewegt, wird zunächst der Forschungsstand zur „Ethnographischen Fußballfanforschung" aufgezeigt und kritisiert. Es gebe eine Einengung auf die Linie Fan - Jugendliche - Männerdomäne - Gewalt - Probleme. Die Geschlechterthematik mit in den Blick zu nehmen, sei durch männliche Involviertheit in das Fantum geprägt: die Beiträge in der Forschung wirken „so, als wolle sich der Autor selbst eine Freude bereiten, indem er zu seinem Hobby auch einmal einen Aufsatz verfasst" (36). Dies geschehe oft im Verbund mit Naturalisierungen von Geschlechterklischees (Gebauer, Sloterdijk, Robson). Reflektierte und diesen Umstand kritisierende Ausnahmen seien bei Bott und Dembowski zu finden. 
Die Geschlechterthematik insgesamt oder Frauen als Fans in den Blick zu nehmen, sei ein neueres Phänomen, etwa bei den Forschungen von Nicole Selmer.

Ein Leitsatz, der den grundlegenden selbstreflexiven Versuch der Autorin in ihrem Werk kennzeichnet, ist die von C. Hagemann-White übernommene Einsicht, „dass das theoretische Wissen um die soziale Konstruiertheit von Geschlecht in die Forschungspraxis umgesetzt werden muss, um zu vermeiden, dass Geschlechterforschung sich in der Falle der Reifizierung verfängt und die Zweigeschlechtlichkeit, die sie eigentlich untersuchen möchte, zugleich reproduziert" (39).

Theoretischen Ausgangspunkt nimmt Sülzle bei der hegemonialen Männlichkeit (Connell), die männliche Herrschaft im Sinne eines dynamischen Systems sieht und „sich über Geschlechterbeziehungen reproduziert“ (50). Innerhalb der Populärkulturen nehme der Fußball eine besondere Stellung ein, er sei mit seiner spezifisch mit ihm verwobenen Männlichkeit eine hegemoniale Sportkultur (114).

Der methodische Zugang erfolgt über die Ethnographie, welche die Involviertheit und die Gefühle der Forscherin nicht nur berücksichtige, sondern zum Potential der Analyse und Erträge mache. Neben der Offenheit allen Daten gegenüber gab es konkrete teilnehmende Beobachtungen von Heim- und Auswärtsspielen der Kickers Offenbach ab dem Jahr 2001 (kontrastierend auch von anderen Mannschaften in anderen Ligen etc.), die in Feldberichten intensiv über zwei Jahre hinweg (und weitere zwei Jahre sporadisch) festgehalten wurden. Darüber hinaus wurden 11 Leitfaden-Interviews geführt, deren Transkripte neben den Feldprotokollen als Datengrundlage gelten.

Almut Sülzle schildert ihre Gefühle gegenüber ihrem Forschungsfeld als ein Wechseln von Begeisterung und Ablehnung. So sei sie fasziniert von der rücksichts- und verantwortungsvollen Zusammengehörigkeit innerhalb der Fangemeinschaft einerseits und abgestoßen von der Gewaltverherrlichung, den Blut-und-Ehre-Sprüchen oder dem exzessiven Alkoholkonsum andererseits (66). Sülzle hat eine klare, sich einfühlende und mitlebende ethnographisch-wissenschaftlich distanzierte Haltung - zumindest dem männlichen Teil der Fans gegenüber. Für den weiblichen Teil der Fans und ihren Aktivismus gegen den Sexismus in der Fußballfankultur stellt sie sich selbst die Frage nach dem going native (70).

Im Ergebnis ihrer substantiellen ethnographischen Untersuchung kommt Sülzle zu folgendem Befund: Bereitschaft zu oder Toleranz der Gewalt gegenüber sei konstitutiv für die Gemeinschaft der Fans und abgrenzend den gesellschaftlichen Normen gegenüber: „Ihre Inszenierung einer ,gewaltigen Stimmungc, das Aufbegehren gegen Grenzziehungen und gegen Polizei und die Selbstdarstellung als Pöbel sind ein Vergnügen, das Frauen wie Männer anzieht" (197).

Auch hier hat die Autorin durch ihre Ethnographie tiefere Einsichten in das Feld, die ihr die Mechanismen der Gewaltfaszination verstehen lassen helfen, ohne dass sie dabei alle Bereiche teilen muss. Direkte körperliche Gewalt sei im höchsten Maße geschlechtlich besetzt: im männlichen Schlägerrausch würde unterschieden und die Gewalt ausschließlich gegen Männer gerichtet. Zu diesen exklusiv männlichen Bereichen der Fußballfankultur würden, beispielsweise auf Auswärtsfahrten im Bus, Themen männlicher Vergemeinschaftung wie Entgrenzung (z. B. Männerstriptease), Alkohol und Sexualität gehören (233).

Es ließe sich eine zweiwertige Dominanz feststellen: Zum einen die Männlichkeitsinszenierung, die in ihrer Übertreibung - ihre Grammatik offen präsentierend - die Grundstrukturen habituell auffrische und in einem gesicherten Raum das zeige und praktiziere, was andernorts (Beruf, Familie etc.) nicht mehr möglich sei. Diese Hypermännlichkeit 
(kernig-kerlige Fanmännlichkeit) nehme auch karnevaleske Züge an (237). Zum anderen sei da die Vergemeinschaftung als zentraler Aspekt des echten Fanseins, die sich in Geborgenheit, Vertrauen, Schutz und gegenseitiger Hilfe der Fan-Familienmitglieder zeige und gegen den Zeitgeist von Kommerz und Konsum gerichtet - sinnstiftend sei (239).

Diese Aufführung ,echter Männlichkeit' und Vergemeinschaftung zur Fanfamilie, die prinzipiell geschlechts- und herkunftsneutral sei, schaffe eine spezielle Anziehungskraft für Fan und Forscherin: „Fankultur folgt einer männlichen Grammatik und ist frauenfeindlich. Gleichzeitig ist die Fankultur für weibliche Fans aber ein Zuhause, wo sie ihre Liebe zum Fußball und zum Verein leben können“"(347).

Unter der männlichen Grammatik der Fankultur fasst die Autorin drei Regeln zusammen: Erstens gelte grundsätzlich, dass Fußball männlich sei, alles Weibliche (und Schwule) würde abgelehnt. Regel zwei besagt, dass die ernsten Spiele nur von den Männern in ritualisierten Rivalitäten (durchaus laut und aggressiv „mit allen Mitteln ,die Ehre“ (348) verteidigen) ausgetragen würden. Die dritte Regel setzt Geschlechtszuschreibungen als hierarchisierende Bewertungen fest, alles Weibliche gelte als Abwertung, die rosa Zicken würden ebenso von den weiblichen Fans wie von den männlichen verachtet.

Die partielle Offenheit der Fankultur für Frauen sei auch in ihrer ironischen Überbetonung der Männlichkeit zu suchen, was den spaßigen Reiz dieser Unternehmung ausmache. Den Zugang würden Frauen über den Grundwert des ,echten Fans' und der Fanfamilie finden, da es kein Modell für Weiblichkeit oder einer Ergänzung von Geschlechtlichkeit gebe. Dies sei auch dafür verantwortlich, dass es keine weitere Geschichte über Frauen zu erzählen gebe, sie seien Fans. Der „männliche Raum der Fankultur wird von Männern und Frauen gemeinsam hergestellt", bestehender Sexismus erschwere zwar teilweise den $\mathrm{Zu}$ gang zu allen Bereichen (353), für Frauen sei die Fankultur aber ein „Ort, an dem sie sich eine Auszeit vom weiblichen Habitus nehmen können, denn das fankulturelle Kapital wird nicht am Körper und auch nicht am Geschlechtskörper festgemacht“ (354).

„Fußball, Frauen, Männlichkeiten“ von Almut Sülzle ist eine tiefgehende (selbst-)reflexive Ethnographie des Fantums, die einem vermittelt, dass die Dimension der Geschlechtlichkeit, also die Aufführung ,echter Männlichkeit', ein Teil dieses Feldes ist, der nicht durch den werturteilend-forschenden Eifer der Autorin hineingetragen wird. Unter dem Gesichtspunkt von Vergemeinschaftungsprozessen fallen gerade die beiden zentralen Ergebnisse als zu unterscheidende Phänomene in das Blickfeld: Männer-Vergemeinschaftung in der überhöhten Männlichkeitsinszenierung und vor allem die geschlechtsirrelevante Vergemeinschaftung der echten Fans. Im Rahmen ihrer Arbeit hat Almut Sülzle diese Befunde zwar nicht an eine Gemeinschaftssoziologie angebunden, gleichsam liegen hiermit anschlussfähige empirisch dichte Analysen vor, die es künftig zu berücksichtigen gilt. Gerade auch, wenn man in den Bereich der Fußballfans intensiver einsteigt, wie in den folgenden Beiträgen.

Ultras sind in der öffentlichen Wahrnehmung zurzeit möglicherweise die am meisten beachtete Fangruppierung überhaupt. So ist es nicht verwunderlich, dass auch das wissenschaftliche Interesse zu diesem Thema steigt. Die Ultras lassen sich begrifflich innerhalb des Fußball-Fanspektrums vermutlich am besten zwischen den kritischen Fans (König 2002: 49ff.) und den erlebnisorientierten Fans einordnen, die neben den konsumorientierten und fußballzentrierten Fans (alle drei bei Heitmeyer 1992) die Stadien bevölkern. Seit der kleineren Studie von Schwier (Schwier 2005), der größeren Untersuchung von Pilz et al. (Pilz, Behn, et al. 2006) sowie der Arbeit von Brenner (Brenner 2009) und der Di- 
plomarbeit von Sommerey (Sommerey 2010) liegen nun aktuelle empirische Forschungsergebnisse zum Thema Ultras vor, die im Folgenden besprochen werden.

Das Werk „Block X - Unter Ultras“ von Sven Kathöfer und Jochem Kotthaus ist als Fachbuch bei Beltz Juventa 2013 erschienen. Im ersten Kapitel (Kathöfer, Kotthaus und Jewgeni Priluzki) geben die Autoren Einblick in ihr Forschungsvorhaben. Es ist geprägt von der Absicht, die Ultras als Lebenswelt unabhängig von den medial und durch Akteure wie Politik, DFL, DFB und anderen Vereins- und Ordnungsinstanzen hervorgebrachten Vorurteilen bzw. Leitthemen zu erforschen und in ihrer eigenen Struktur zu rekonstruieren. Dies ist als unabhängige sozialwissenschaftliche Grundlagenforschung zu verstehen, ohne Drittmittel und Feldkontakte „von oben“. Dieser qualitative Ansatz wird mit einem Methodenmix qualitativer Verfahren wie 55 Interviews (biographisch-narrative, Feldgespräche, Leitfadeninterviews), 11/2 Jahre Feldaufenthalten (Beobachtung, teilnehmende Beobachtung) und verschiedenen Auswertungsverfahren (kodierend (konzeptbildend) nach der Grounded Theory, Inhaltsanalyse (Mayring) und Fallanalysen) durchgeführt. Auf der einen Seite ist dies ein anspruchsvoller, vielseitiger Ansatz, der über die Verwendung möglichst vieler Verfahren eine möglichst große Authentizität herstellen möchte - auf der anderen Seite stellt sich die Frage nach der Vereinbarkeit einer solchen Methoden- und Analyse-Triangulation, etwa den Unterschieden von fallorientierten Analysen einerseits und konzeptbildenden Analysen (G.T.) andererseits, oder inhaltszusammenfassenden Verfahren und Verfahren zur tieferen Analyse.

Das zweite Kapitel (Kathöfer, Kotthaus und Martin Willmann) widmet sich der historischen Entwicklung von Fußball, seinen Fans sowie dem Stand der Forschung. Nach Heitmeyer / Peter wird unterschieden in konsumorientierte, fußballzentrierte und erlebnisorientierte Fans, die nach Giulianotti und König hinsichtlich der kritischen Fans erweitert werden. Als dominante Gruppe seien heutzutage die Ultras zu bezeichnen, bekannt und als Gruppe auffällig seien aber auch die in ihrer Bedeutung sinkenden Kuttenfans und Hooligans zu nennen. Eine weitere Kategorisierung von Fans finde durch die Polizei unter dem Gesichtspunkt der Gewaltbereitschaft statt: A (friedlich), B (gewaltbereit/-geneigt) und $\mathrm{C}$ (gewaltsuchend).

In Kapitel drei (Kathöfer und Kotthaus) stellen die Autoren drei Entwicklungsphasen der Ultraszene vor: die Konstituierungsphase, die Etablierungsphase und die Wandlungsphase. Als italienischer Import und mit dem Retter-Mythos der Fans insgesamt, mussten sich die Ultras in der Konstituierungsphase zunächst gegen die etablierten Fans - durchaus auch territorial in den Stadien verstanden - durchsetzen. Die Etablierungsphase sei durch Zeigen von Präsenz, Choreographien und dem „Spiel“ um die Zaunfahne (mit ähnlicher Bedeutung wie in mittelalterlichen Gefechten) gekennzeichnet. In der Wandlungsphase schließlich würden die (teilweise durch die Vereine bereitgestellten) eigenen Räumlichkeiten der Ultras und deren größere Offenheit nach Außen an Gewicht gewinnen. Das habe auch zur Folge, dass das Leben in der Szene zum Konsumgut werden könne, dass es innere Distinktionen mit Aufspaltungen und Neugründungen gebe.

Kapitel vier (Richard Göldner, Kathöfer und Kotthaus) untersucht die Lebenswelt der Ultras. Die Szeneeinstiege würden zumeist über einen bereits existierenden familiären fußballbezogenen Hintergrund, einen solchen der Peergroup oder einen sportlichen laufen. Gelegentlich fänden sie auch als Abgrenzungshandlung statt, ohne Fußballaffinität ginge aber nichts. Die Autoren differenzieren drei Gruppengrößen: kleine Gruppierungen mit bis zu 50 Personen, mittlere mit bis zu 150 Personen und Gruppengrößen darüber. Bei 
der Vereinswahl spiele dessen Präsenz im sozialen Raum, der Region und sein Prestige eine wichtige Rolle. Die Einsteiger gelängen in hierarchische Strukturen, die durch den sehr wesentlichen Aspekt der Leistung für die eigene Gruppe und dem daraus gewonnenen Prestige strukturiert würden (mit vereinstypischen Leistungspositionen). Auch wenn es Verhaltensvorgaben innerhalb der Gruppen gebe, sind diese bei extrem gering funktionierenden Sanktionsmechanismen nicht vollständig umgesetzt. Trotz gemeinsamer Kommerzkritik existiere Uneinigkeit über ihre konkrete Ausgestaltung.

In Kapitel fünf widmen sich die Autoren (Kathöfer und Kotthaus und Priluzki) den Konfliktfeldern der Ultras und ihrer medialen Vermittlung. Gruppenbezogene Menschenfeindlichkeit (Heitmeyer) fände sich - wie in der gesamten Fanszene - auch bei den zumeist jungen männlichen Ultras. So seien rassistische, fremdenfeindliche und antisemitische sowie homophobe Äußerungen diskriminierender Art Teil der sogenannten „Beschimpfungskultur“, und Gewalterfahrung sei Teil der Lebenswelt von Ultras. Medial werde dieser Umstand oft auf die Ultras „verengt“ dargestellt, was zu einem ambivalenten Verhältnis führe. Politische Positionierungen der Ultras würden aber vermieden oder seien umstritten, sofern sie stattfinden.

Kapitel sechs (Göldner, Kathöfer und Kotthaus) befasst sich mit den inneren Strukturen der Ultras als Jugendszene, die in jeweils eigenen Fallgeschichten nachvollziehbar ausgeführt werden. Es gebe im Zentrum den harten Kern, der aus Initiatoren, Idealisierten und Subkulturellen bestehe, die allesamt kreative und organisatorische, immer zeitintensive Aufgabenbereiche übernehmen. In der Konvektionszone befänden sich die Balancierer und Anwärter, die ständig in einer Strömungsbewegung zwischen innen und außen seien, vom harten Kern energetisiert, von außen (Beruf, Familie etc.) wieder abgekühlt, nur um wieder von vorne zu beginnen. In der Korona schließlich befänden sich die Gruppenlosen (z. B. ehemalige Mitglieder), die Freerider und die Personen im Umfeld (intern auch „Modeultras“ oder „Konsumultras“ genannt) sowie die Auffälligen, das seien die "gewaltoffenen" Fans (zuweilen auch zu Hooligans abwandernd), deren delinquente Aktivitäten auch das Bild der Ultras in den Medien prägen würden.

Das Werk hält insgesamt, was es verspricht und liefert dem Leser einen an den Stimmen und Relevanzstrukturen der Akteure orientierten Einblick in die Ultra-Fanszene, der nicht vergisst, die für die Wissenschaft und Öffentlichkeit interessanten Fragen systematisch zu behandeln. Die Fortentwicklung - oder feldspezifische Anpassung - vorhandener Konzepte (vgl. das Kern / Korona / Konvektionszonen-Bild (172) mit einem Organisationseliten-Bild (Hitzler und Niederbacher 2010: 23)) erweitert eine gemeinschaftssoziologische Sichtweise und stellt einen wichtigen Beitrag dar.

Gerade (2013) in seiner 5. erweiterten Auflage im PapyRossa-Verlag erschienen, zeigt das Werk von Jonas Gabler („Die Ultras - Fußballfans und Fußballkulturen in Deutschland“) einen - zwar etwas weniger an wissenschaftlichen, denn an gesellschaftsaufklärenden Prinzipien orientierten - fundierten Einblick in die historisch-internationale Entwicklung und derzeitige Lage der Ultras in Deutschland. Als Hauptquelle seiner Analysen dient dem Autor nach eigenen Angaben das Ultra Fanzine „Blickfang Ultrà“.

Gabler geht zunächst auf das Phänomen der Ultraszene als eine überwiegend männliche Jugendkultur ein, in der das Streben nach Normierungsauflösung in der Adoleszenzphase und das einfache Strukturen bietende Freund/Feind-Schema rituell und experimentell ausgelebt werden könne. Ein Blick auf die Entwicklung der Fankultur in Deutschland schließt sich an, in dem auch die definitorischen Abgrenzungen zu Kuttenfans und Hooli- 
gans vollzogen werden. Nach dem historischen Abriss der Entstehung der Ultras in Italien folgt die Kennzeichnung der Ultraszene in Deutschland mit ihren Organisationsstrukturen, den Ausdrucksformen des Supports und den Beziehungen zu den anderen Gruppierungen. Dem für die Ultras selbst wesentlich identitätsstiftenden Punkt des Widerstandes gegen die Kommerzialisierung im Fußball wird ein volles Kapitel gewidmet und anhand typischer Beispiele (etwa FC Bayern, Hoffenheim und RB Leipzig) erläutert. Die Gewaltund Repressionsfrage bzgl. der Ultras wird als zwar berechtigtes öffentliches Interesse, aber eben auch als eine unzureichende und teilweise unzutreffende Reduktion der Wahrnehmung behandelt. Sie erschwere den Zugang zu den teilweise hochgradig reflektierten und gesellschaftskritisch analysierenden Ultras nur unnötig. In der Frage der politischen Gesinnung sei nach dem Rückgang des Rechtsextremismus in den Stadien eine große Heterogenität zu erkennen, die sich nachrangig des eigentlichen Zweckes - der Unterstützung des Vereins mit allen Kräften - verhalte. So sieht Gabler die Ultraszene auch als eine Art Sozialisationsinstanz, die den Jugendlichen über Anerkennung eine selbstbewusste Persönlichkeitsentwicklung ermögliche.

Das Buch von Gabler hat - aufgrund seiner nicht zu eng wissenschaftlich ausgerichteten Konzeption und Sprache - eine größere Resonanz gefunden und ist aus der öffentlichen und wissenschaftlichen Debatte über das Thema Ultra nicht mehr wegzudenken. Diese „Gelenkfunktion“ sollte nicht unterbewertet werden, wenn sich Soziologie auch als Teil einer gesellschaftsinformierenden Wissenschaft verstehen möchte.

Der 2010 erschienene kleinere Band von Daniel Langer („Faszination Ultras - Aspekte und Erklärungsansätze zur Fußballfan- und Jugendkultur") arbeitet unter sehr ähnlichen Kapiteln wie Gabler die bestehende Literatur systematisch auf, wobei der theoretische Zugang über den Jugendbegriff (als Sozialisationsphase) bzw. den Jugendkulturbegriff vorgenommen wird. Im Ergebnis kommt er zu der Einschätzung, dass Ultras als sinnvolle Sozialisationsinstanz zur Persönlichkeitsfindung gelten könnten (Chance), aber immer mit gleichzeitiger Möglichkeit von Gewalt verwoben seien (Risiko).

Hier ist Langer auf einer Linie mit den anderen hier besprochenen Büchern, die insbesondere von den Medien und institutionellen Vertretern (und damit der von ihr stark beeinflussten Öffentlichkeit) eine deutlichere Relativierung einer unreflektierten Gleichsetzung der Ultraszene mit Gewaltausübung fordern. Es lässt sich ein deutlicher Trend in der Ultra-Forschung markieren: Zum einen wird die mediale und ordnungspolitische Sicht auf das Phänomen Ultra als verengt „entlarvt“, wichtige Binnendifferenzierungen (Entwicklungsprozess, Struktur, kritisch reflektierte Haltung etc.) und die Abgrenzung zu dem Phänomen Hooligan dargelegt. Zum anderen zeigt sich, dass derartige Forschungen in brenzligen Feldern „öffentlicher" Verengung - größtenteils mit qualitativen Methoden vollzogen werden konnten, die durch ihre Nähe an den Relevanzstrukturen der Subjekte und ihre Offenheit in solchen Feldern ihre Stärke zu haben scheinen. Möglicherweise lässt sich dies noch weiter verallgemeinern: In den Feldern einer an Vergemeinschaftungsprozessen interessierten Soziologie, wo es immer zumindest auch um Gruppenidentitätsaufbau (aktiv oder passiv) durch Abgrenzung oder Ausgrenzung geht, ist ein subjektorientierter Feldzugang von besonderem Wert.

Yvonne Niekrenz geht in ihrer 2010 an der Universität Rostock angenommenen und 2011 in der Reihe Erlebniswelten des VS-Verlags erschienenen Dissertation „Rauschhafte Vergemeinschaftungen - Eine Studie zum rheinischen Straßenkarneval“ der Frage nach der begrifflichen und phänomenalen Fassung rauschhafter Vergemeinschaftung nach. Als 
Ausgangsthesen formuliert sie die Annahme, dass rauschhafte Vergemeinschaftungen zum einen eine Grundlage des sozialen Zusammenlebens seien, zum anderen zur Bewältigung vielfältiger Anforderungen an das heutige Individuum und der Endlichkeit menschlichen Lebens beitragen.

Theoretisch nimmt Yvonne Niekrenz den Ausgangspunkt bei Victor Turner (Communitas als undifferenziertes Gegenmodell zur strukturierten Gesellschaft) und Michel Maffesoli (Orgiasmus als Träger des Gemeinschaftslebens). Die Autorin gibt zunächst den Stand der Forschung zu den einzelnen Konzepten der Vergemeinschaftung und des Rausches - auch unter dem Gesichtspunkt einer Vorstrukturierung nach Raum, Zeit und Körper - wieder. Anschließend bringt sie unter dem Begriff rauschhafte Vergemeinschaftung beide zusammen und konkretisiert darunter freiwillige ritualisierte „Gesellungsformen, die (1) körperliche Kopräsenz mit (thematisch) fokussierten Interaktionen, (2) ein Zusammengehörigkeitsgefühl bei den Gruppenmitgliedern im Sinne einer durch das Individuum bestimmten emotionalen Zugehörigkeit, (3) eine deutliche Veränderung des sozialen Handelns in Bezug auf Emotionskontrolle und Konventionen sowie (4) eine kurzzeitig veränderte Wahrnehmung hinsichtlich Raum-Zeit-Bezügen und/oder Körpererfahrungen voraussetzen“ (75).

Eine kurze historische und aktuell informatorische Einführung in den rheinischen Karneval bringt den Leser an das im empirischen Fokus stehende Phänomen als Beispiel von rauschhafter Vergemeinschaftung heran. Die empirische Arbeit ist als Ethnographie angelegt, deren Feldphase zwei Karnevals umfasste, die Saison 2007 und 2008. Neben der teilnehmenden Beobachtung von Festumzügen, Karnevalsfeiern, Sitzungskarnevals und freien Zusammenkünften führte Yvonne Niekrenz narrative und ethnographische Interviews und sammelte zugängliches Datenmaterial der beteiligten Akteure. Die jeweilige Auswahl von Kontrastfällen zur Konzepterstellung wurde gemäß des theoretical samplings der Grounded Theory vollzogen. In den Analyseverfahren ergänzen sich rekonstruktive Hermeneutik und ethnographische Semantikanalyse. Die empirischen Ergebnisse werden entlang der vorstrukturierenden Dimensionen Raum, Zeit und Körper entfaltet:

Für den Raum wird die Gebundenheit an Köln und dortige Orte und Bauten herausgestellt, dies werde auch und gerade in Gesängen deutlich. Die Auflösung normaler funktionaler Nutzungsroutinen der städtischen Bereiche und die Zentrierung der Geschehnisse im Stadt-Zentrum seien eine wesentliche Änderung im Karneval, genauso wie die politische und weltanschauliche Grenzauflösung bei gleichzeitiger strukturell-religiöser Dimension der Aktivitäten durch Ritual und Gemeinschaftsbildung im säkularen Bereich. Aus räumlicher Sicht fänden bereits starke Vergemeinschaftungsprozesse am Ort des HinTransportes (wie etwa dem Zug) statt, in denen die Vorfreude als wesentlicher Motor gelte.

In der Dimension der Zeit würden klare Bezugspunkte für das Austreten aus den alltäglichen und gleichförmigen Temporalstrukturen, sowie innerhalb des Karnevals eine deutlich zeitliche Programmstruktur gelten. Die zyklische Wiederkehr reproduziere Erinnerung an letzte Karnevals, biete eine zeitliche Konstante in Biographie und Familiengeschichte und erinnere an das Fortdauern der Welt, so wie die begrenzte lineare Zeit des Karnevals selbst an die Endlichkeit des eigenen Seins erinnere.

Die körperliche Dimension zeige sich wie folgt: Körperliche Gebundenheit von Kostüm, Maske und Verkleidung würden als vergemeinschaftender Übertritt und Eintritt in die andere Welt fungieren. Emotionen als wesentlicher körperlicher Bestandteil gelten konstitutiv für den Karneval, d.h. gute Stimmung, Feierlaune und gut drauf Sein. Tanzen 
und außeralltägliche Körperordnungen wie beispielsweise das Schunkeln demonstrieren eine kollektive, oft mimetisch synchronisierte Orientierung der Jecken. Alkohol und der damit zusammenhängende Rausch seien fester Bestandteil der rauschhaften Vergemeinschaftung im Karneval, sie seien Garant für den gemeinschaftlich ritualisierten Übertritt ins Außeralltägliche. Hiermit verbunden - tendenziell eher bei den Jugendlichen - seien normüberschreitende, enthemmte körperliche Aktivitäten in Richtung von Gewalt und Sexualität, die für den erfahrenen Jecken keine zentrale und eher überspitzt aufgeführte Rolle denn ausgeführte Realität bedeute.

Das Karnevaltypische an der rauschhaften Vergemeinschaftung sei nun, dass fußend auf den (traditionellen) gemeinschaftlichen Verbindungen der Familie, Freunde/Bekannte, Nachbarn, Kollegen/Geschäftspartner und Vereinsfreunde diese zum einen zusammen und zum anderen vor allem mit Fremden unter dem Dach der Karneval-Idee verbunden würden. Dieses, unter der beschriebenen raumzeitlichen Kopräsenz des Körperlichen, ließe den Karneval zur rauschhaften Vergemeinschaftung werden.

Einem Drama (Turner) gleich durchlaufe der Karneval Bruch, Krise, Bewältigung und positive Wiederherstellung der Alltagsordnung - diese rauschhafte Vergemeinschaftung gelte somit als Kitt für das soziale Gefüge, auch wenn dem gespielten Bruch die Potentialität eines echten Bruches innewohne. Zugleich wirke die rauschhafte Vergemeinschaftung als Bewältigungsstrategie aktueller gesellschaftlicher Anforderungen an das Individuum sowie für die generelle Endlichkeit menschlicher Existenz.

Den großen Gewinn dieser Arbeit sehe ich in der theoretischen Konzeptualisierung und empirischen Begründung des - für eine Soziologie der Gemeinschaft und in dieser Tiefe - neuartigen Begriffs der rauschhaften Vergemeinschaftung. Gerade die in der Serie Erlebniswelten des VS-Verlags verhandelten Phänomenbereiche, die oft unter dem Aspekt der Events gefasst werden, erhalten hiermit einen differenzierteren Begriffsapparat, der die Soziologie der Gemeinschaft voranbringen wird. Insbesondere erweitert er das Verständnis, dass der Karneval nicht nur einen traditionalen Vergemeinschaftungsprozess darstellt und dass Events nicht nur posttraditionale Elemente haben, wie es zuweilen gesehen wird. Die Autorin muss sich allerdings folgende Frage gefallen lassen: Ist es gelungen zu erklären, was das besonders „postmoderne“ am heutigen Karneval sein soll? Möglicherweise ist der Begriff der rauschhaften Vergemeinschaftung nicht so nahe dem der posttraditionalen Vergemeinschaftungsformen, wie die Autorin es darzustellen sucht. Das Neue kann also in der Ergänzung der traditionalen Gemeinschaftsbezüge des Karnevals um die rauschhaften Vergemeinschaftungsprozesse gesehen werden, die wiederum Attraktivität für Akteure posttraditionaler Vergemeinschaftungsprozesse ausstrahlen. Denn die traditionalen - eben festen - Sozialbeziehungen wie Vereine etc. stabilisieren den rituellen Rahmen, der für die Wiederholung des Aktes der Vergemeinschaftung beim Karneval nötig ist (252). Insofern ist $u$. U. auch ein verbindendes Konzept entwickelt worden, was merkwürdig getrennte Bereiche soziologischer Gemeinschaftsforschung verbinden kann.

Was lässt sich nun für ein Resümee der hier besprochenen Bücher zu Fans und Vergemeinschaftungsprozessen ziehen? Zum einen haben sich das Phänomenspektrum selbst sowie die methodischen und theoretischen Zugänge erweitert (vgl. Roose, Schäfer et al., Nern). Zum anderen zeigen sich gegenstandsnahe Binnendifferenzierungen, die - zumeist über qualitative Zugänge ermöglicht - neue Blickwinkel aufzeigen und zur Auflösung verhärteter Mediendeutungen beitragen können (Kathöfer / Kotthaus, Gabler, Langer und 
Sülzle). $\mathrm{Zu}$ diesen Binnendifferenzierungen gehören etwa die Unterscheidung von echten (genderfreien) Fans und Männervergemeinschaftungsprozessen im Konglomerat Fans (vgl. Sülzle), aber auch die von traditionalen und posttraditionalen Elementen gerahmte rauschhafte Vergemeinschaftung (vgl. Niekrenz) oder die für das Phänomen Fan gleichsam konstitutiven Beziehungsbewegungen konjunktiver und disjunktiver Art (vgl. Fritzsche). Gerade die empirisch gewonnenen begrifflichen Verbesserungen können als besonderer Gewinn geltend gemacht werden.

\section{Literatur}

Brenner, David (2009): Neues aus der Fankurve. Wie Ultras und andere Fanszenen die Fankultur verändern. Marburg: Tectum.

Etzioni, Amitai (1995): Die Entdeckung des Gemeinwesens. Ansprüche, Verantwortlichkeiten und das Programm des Kommunitarismus. Stuttgart: Schäffer-Poeschel.

Grundmann, Matthias / Dierschke, Thomas et al. (Hrsg.) (2006): Soziale Gemeinschaften. Experimentierfelder für kollektive Lebensformen. Münster: LIT.

Heitmeyer, Wilhelm (1992): Die unterschiedlichen Fan-Gruppierungen. In: Heitmeyer, Wilhelm und Peter, Jörg-Ingo (Hrsg.), Jugendliche Fußballfans. Soziale und politische Orientierungen, Gesellungsformen, Gewalt. Weinheim: Juventa, S. 56-63.

Hitzler, Ronald / Niederbacher, Arne (2010): Leben in Szenen. Formen juveniler Vergemeinschaftung heute. Wiesbaden: VS.

Joas, Hans (2007): Städte, Gemeinden und Urbanisierung. In: Joas, Hans (Hrsg.), Lehrbuch der Soziologie. Frankfurt a. M.: Campus, S. 598-604.

König, Thomas (2002): Fankultur - Eine soziologische Studie am Beispiel des Fußballfans. Münster: LIT.

Miner, Horace (1956): Body Ritual among the Nacirema. In: American Anthropologist 58 (3), S. 503-507.

Pilz, Gunter A. / Behn, Sabine et al. (Hrsg.) (2006): Wandlungen des Zuschauerverhaltens im Profifußball. Schorndorf: Hofmann.

Schwier, Jürgen (2005): Die Welt der Ultras. Eine neue Generation von Fußballfans. In: Sport und Gesellschaft 2 (1), S. 21-38.

Sommerey, Marcus (2010): Die Jugendkultur der Ultras - Zur Entstehung einer neuen Generation von Fußballfans. Stuttgart: ibidem.

Tönnies, Ferdinand (1991): Gemeinschaft und Gesellschaft. Grundbegriffe der reinen Soziologie. Darmstadt: WB. 